\title{
Measure of Lattice-Valued Direct $F$-transforms and Its Topological Interpretations
}

\author{
Anand Pratap Singh $\left.{ }^{(}\right)(\mathbb{D}$ and Irina Perfilieva \\ Institute for Research and Applications of Fuzzy Modeling, CE IT4Innovations, \\ University of Ostrava, 30. dubna 22, 70103 Ostrava 1, Czech Republic \\ \{anand.singh, irina.perfilieva\}@osu.cz
}

\begin{abstract}
The goal is to introduce and study the measure of quality of approximation of a given fuzzy set by its lattice-valued $F$-transform. Further, we show that this measure is connected with an Alexandroff $L M$-fuzzy topological (co-topological) spaces. Finally, we discuss the categorical relationship between the defined structures.
\end{abstract}

Keywords: $M$-valued partition $\cdot$ Direct $F$-transforms $\cdot$ Fuzzy inclusion measure $\cdot L M$-fuzzy (co)topology $\cdot$ Ditopology

\section{Introduction}

The importance of various kinds of transforms such as Fourier, Laplace, integral, wavelet are well-known in classical mathematics. The main idea behind these techniques consists of transforming an original space of functions into a new computationally simpler space. Inverse transformations back to the original spaces and produce either the original functions or their approximations. The notion of $F$-transforms were proposed in [17] has now been significantly developed. Through the viewpoint of application purpose, this theory represent new methods which have turned out to be useful in denoising, time series, coding/decoding of images, numerical solutions of ordinary and partial differential equations (cf., $[3,13,24])$ and many other applications.

In the seminal paper [17], $F$-transforms were defined on real-valued functions and another type of $F$-transform was also introduced based on a residuated lattice in the interval $[0,1]$. A number of researchers have initiated the study of $F$-transforms, where they are applied to $L$-valued functions in a space defined by $L$-valued fuzzy partitions (cf., $[15,16,18,19,25])$, where $L$ is a complete residuated lattice. Among these studies, a categorical study of $L$-partitions of an arbitrary universe is presented in [15] and an interesting relationship among $F$-transforms, $L$-topologies/co-topologies and $L$-fuzzy approximation spaces are established in [19]. The relationships between $F$-transforms and similarity relations are investigated in [16], axiomatic study of $F$-transforms have been done in [14], while $F$-transforms based on a generalized residuated lattice are studied in [25]. 
In the past few years, some studies have been conducted on the theoretical development of lattice-valued $F$-transforms. Among them, the papers [1820,22] are focused on establishing the relationship between the lattice-valued $F$-transform and various structured spaces, namely, fuzzy (co) topological/pre(co)topological space, fuzzy approximation space and fuzzy interior/closure spaces. The ground structure for the above-mentioned studies is the lattice-valued $F$-transform defined on a space with a fuzzy partition [19]. There are many papers $[2,12,28]$, where implications are used to evaluate the measure of inclusion in the same lattice $L$. To our knowledge, the first attempt to measure the degree of roughness of a given fuzzy set in fuzzy rough set theory was undertaken in $[6,7]$. In $[7]$, an approach to measure the quality of rough approximation of fuzzy set is discussed. Motivated by this work, we aim at studying the measure of approximation by the lattice-valued direct $F$-transforms. In other words, we measure the degree of inclusion of lattice-valued $F$-transforms into the $L$-fuzzy set.

For this purpose, in Sect.3, we consider a more general version of latticevalued $F$-transform defined on space with an $M$-valued partition. Specifically, we define the lattice-valued $F$-transform operators of an $L$-fuzzy subset of a set endowed with an $M$-valued partition. These operators, as special cases, contain various rough approximation-type operators used by different authors $[10,11,21,26]$. Interestingly, as a main result of this contribution, in Sect. 4 , we show that the $M$-valued measure of $F$-transform operators based on space with $M$-valued partition determine the Alexandroff $L M$-fuzzy topological (cotopological) spaces. We also discuss the mentioned relationship through a categorical viewpoint. It is worth mentioning that our work differs from the existing study in the sense that here we consider the lattice-valued $F$-transform operator based on the $M$-valued partition represented by $M$-fuzzy preorder relation and showed that with this $M$-fuzzy preorder relation, Alexandroff $L M$-fuzzy topology (co-topology) can be induced by defining the $M$-valued measure of direct $F$-transforms. Finally, we give some direction for further research.

\section{Preliminaries}

Here, we recall the basic notions and terminologies related to residuated lattices, integral commutative cl-monoid, measure of inclusion between two fuzzy sets and fuzzy topological spaces. These terminologies will be used in the remaining text.

Definition 1 [1,8,9]. An integral commutative cl-monoid (in short, iccl monoid) is an algebra $(L, \leqslant, \vee, \wedge, \otimes)$ where $(L, \leqslant, \vee, \wedge)$ is a complete lattice with the bottom element $0_{L}$ and top element $1_{L}$, and $\left(L, \otimes, 1_{L}\right)$ is a commutative monoid such that binary operation $\otimes$ distributes over arbitrary joins, i.e.,

$$
a \otimes\left(\vee_{i \in I} b_{i}\right)=\vee_{i \in I}\left(a \otimes b_{i}\right), \text { for all } a \in L,\left\{b_{i} \mid i \in I\right\} \subseteq L .
$$

Given an iccl-monoid $(L, \leqslant, \vee, \wedge, \otimes)$, we can define a binary operation " $\rightarrow$ ", for all $a, b, c \in L$,

$$
a \rightarrow b=\vee\{c \in L \mid c \otimes a \leq b\}
$$


which is adjoint to the monoidal operation $\otimes$, i.e.,

$$
a \otimes b \leq c \Longleftrightarrow a \leq b \rightarrow c .
$$

A particular example of residuated lattice is Lukasiewicz algebra

$$
\mathscr{L}_{L}=([0,1], \vee, \wedge, \otimes, \rightarrow, 0,1),
$$

where the binary operations " $\otimes$ " and " $\rightarrow$ " are defined as, $a \otimes b=\min (a+b-1,1)$ and $a \rightarrow b=\max (1-a+b, 0)$.

The following properties of residuated lattice will be used, for proof refer to $[1,5,9]$.

Proposition 1. Let $(L, \leqslant, \wedge, \vee, \otimes, \rightarrow)$ be a residuated lattice. Then for all $a, b, c \in L$,

(i) $a \leq b \rightarrow(a \otimes b), a \otimes(a \rightarrow b) \leq b$,

(ii) $a \otimes(b \rightarrow c) \leq(a \rightarrow b) \rightarrow c$,

(iii) $a \otimes\left(\vee_{j \in J} b_{j}\right)=\vee_{j \in J}\left(a \otimes b_{j}\right)$,

(iv) $a \otimes\left(\wedge_{j \in J} b_{j}\right) \leq \wedge_{j \in J}\left(a \otimes b_{j}\right)$,

(v) $\vee_{j \in J}\left(a_{j} \rightarrow b\right) \leq \wedge_{j \in J} a_{j} \rightarrow b$

(vi) $a \rightarrow(b \rightarrow c)=(a \otimes b) \rightarrow c=b \rightarrow(a \rightarrow c)$,

(vii) $a \rightarrow \wedge_{j \in J} b_{j}=\wedge_{j \in J}\left(a \rightarrow b_{j}\right)$,

(viii) $\vee_{j \in J} a_{j} \rightarrow b=\wedge_{j \in J}\left(a_{j} \rightarrow b\right)$,

(ix) $a \rightarrow \vee_{j \in J} b_{j} \geq \vee_{j \in J}\left(a \rightarrow b_{j}\right)$

Given a nonempty set $X, L^{X}$ denotes the collection of all $L$-fuzzy subsets of $X$, i.e. a mapping $\lambda: X \rightarrow L$. Also, for all $a \in L, \mathbf{a}(x)=a$ is a constant $L$-fuzzy set on $X$. The greatest and least element of $L^{X}$ is denoted by $1_{X}$ and $0_{X}$ respectively. For all $\lambda \in L^{X}$, the $\operatorname{core}(\lambda)$ is a set of all elements $x \in X$, such that $\lambda(x)=1$. An $L$-fuzzy set $\lambda \in L^{X}$ is called normal, if core $(\lambda) \neq \emptyset$.

The notion of powerset structures are well known and have been widely used in several constructions and applications. According to Zadeh's principle, any map $f: X \rightarrow Y$ can be extended to the powerset operators $f \rightarrow: L^{X} \rightarrow$ $L^{Y}$ and $f^{\leftarrow}: L^{Y} \rightarrow L^{X}$ such that for $\lambda \in L^{X}, \mu \in L^{Y}, y \in Y, f^{\rightarrow}(\lambda)(y)=$ $\vee_{x, f(x)=y} \lambda(x), f^{\leftarrow}(\mu)=\mu \circ f$.

Definition 2. [4] Let $X$ be a nonempty set. Then for given two L-fuzzy sets $\lambda, \mu$ and for each $x \in X$, following are the new $L$-fuzzy sets defined:

$$
\begin{gathered}
\lambda=\mu \Longleftrightarrow \lambda(x)=\mu(x), \quad \lambda \leq \mu \Longleftrightarrow \lambda(x) \leq \mu(x), \\
(\lambda \wedge \mu)(x)=\lambda(x) \wedge \mu(x), \quad(\lambda \vee \mu)(x)=\lambda(x) \vee \mu(x), \\
(\lambda \otimes \mu)(x)=\lambda(x) \otimes \mu(x),(\lambda \rightarrow \mu)(x)=\lambda(x) \rightarrow \mu(x) .
\end{gathered}
$$


Let $I$ be a set of indices, $\lambda_{i} \in L^{X}, i \in I$. The meet and join of elements from $\left\{\lambda_{i} \mid i \in I\right\}$ are defined as follows:

$$
\left(\wedge_{i \in I} \lambda_{i}\right)(x)=\wedge_{i \in I} \lambda_{i}(x),\left(\vee_{i \in I} \lambda_{i}\right)(x)=\vee_{i \in I} \lambda_{i}(x) .
$$

In this paper, we work with two independent iccl-monoids $L=\left(L, \leqslant, \vee, \wedge, \otimes_{L}\right)$ and $M=\left(M, \leqslant, \vee, \wedge, \otimes_{M}\right)$. The iccl-monoid $L$ is used as range for $L$-fuzzy sets (i.e. for the approximative objects), while $M$ is used as the range of values taken by the measure estimating the precision of the approximation. Both the iccl-monoids are unrelated, however for proving some results based on measure of approximation, a connection between them is required, for this purpose we define fixed mappings $\phi: L \rightarrow M$ and $\psi: M \rightarrow L$ such that the top and bottom elements are preserved i.e., for $\left\{a_{i} \mid i \in I\right\} \subseteq L, \quad\left\{b_{i} \mid i \in I\right\} \subseteq$ $M, \phi\left(\wedge_{i \in I} a_{i}\right)=\wedge_{i \in I} \phi\left(a_{i}\right), \psi\left(\vee_{i \in I} b_{i}\right)=\vee_{i \in I} \psi\left(b_{i}\right)$. Additionally, we need that $\phi\left(\alpha \otimes_{L} a\right)=\phi(\alpha) \otimes_{L} \phi(a), \forall \alpha, a \in L$ and $\psi\left(\beta \otimes_{M} b\right)=\psi(\beta) \otimes_{M} \psi(b), \forall \beta, b \in M$.

Now we recall the following definition of $M$-fuzzy relation from [27].

Definition 3. [27] Let $X$ be a nonempty set. An $M$-fuzzy relation $\delta$ on $X$ is an $M$-fuzzy subset of $X \times X$. An $M$-fuzzy relation $\delta$ is called

(i) reflexive if $\delta(x, x)=1 \forall x \in X$,

(ii) transitive if $\delta(x, y) \otimes \delta(y, z) \leq \delta(x, z), \forall x, y, z \in X$.

$A$ reflexive and transitive $M$-fuzzy relation $\delta$ is called an $M$-fuzzy preorder.

Here, we define the measure of inclusion between two given $L$-fuzzy sets as it was introduced in [7].

Definition 4. Let the iccl-monoids $L, M$ be given and $\phi: L \rightarrow M$ be the fixed mapping. The $M$-valued measure of inclusion of the L-fuzzy set $\lambda$ into the L-fuzzy set $\mu$ is a map $\hookrightarrow: L^{X} \times L^{X} \rightarrow M$ defined as

$$
\lambda \hookrightarrow \mu=\wedge_{x \in X} \phi(\lambda(x) \rightarrow \mu(x)), \text { for all } \lambda, \mu \in L^{X} .
$$

In other words, the measure of inclusion function $\hookrightarrow$ can be defined by $\lambda \hookrightarrow$ $\mu=\phi(\wedge(\lambda \rightarrow \mu))$, where the infimum of the L-fuzzy set $\lambda \hookrightarrow \mu$ is taken in the lattice $L^{X}$.

Below, we give some useful properties of the map $\hookrightarrow$, which is similar (in some sense) to the properties of residuated lattices.

Proposition 2. [7] The inclusion map $\hookrightarrow: L^{X} \times L^{X} \rightarrow M$ satisfies the following properties.

(i) $\left(\vee_{i \in I} \lambda_{i}\right) \hookrightarrow \mu=\wedge_{i \in I}\left(\lambda_{i} \hookrightarrow \mu\right), \forall\left\{\lambda_{i} \mid i \in I\right\} \subseteq L^{X}, \mu \in L^{X}$,

(ii) $\lambda \hookrightarrow\left(\wedge_{i \in I} \mu_{i}\right)=\wedge_{i \in I}\left(\lambda_{i} \hookrightarrow \mu\right), \forall \lambda \in L^{X},\left\{\mu_{i} \mid i \in I\right\} \subseteq L^{X}$,

(iii) $\lambda \hookrightarrow \mu=1 \Leftrightarrow \lambda \leq \mu$,

(iv) $1_{X} \hookrightarrow \lambda=\phi\left(\wedge_{x} \bar{\lambda}(x)\right), \forall \lambda \in L^{X}$,

(v) $\lambda \hookrightarrow \mu \leq(\lambda \otimes c \hookrightarrow \mu \otimes c), \forall \lambda, \mu, c \in L^{X}$, 
(vi) $\left(\wedge_{i} \lambda_{i}\right) \hookrightarrow\left(\wedge_{i} \mu_{i}\right) \geq \wedge_{i}\left(\lambda_{i} \hookrightarrow \mu_{i}\right), \forall\left\{\lambda_{i} \mid i \in I\right\},\left\{\mu_{i} \mid i \in I\right\} \subseteq L^{X}$, (vii) $\left(\vee_{i} \lambda_{i}\right) \hookrightarrow\left(\vee_{i} \mu_{i}\right) \geq \wedge_{i}\left(\lambda_{i} \hookrightarrow \mu_{i}\right), \forall\left\{\lambda_{i} \mid i \in I\right\},\left\{\mu_{i} \mid i \in I\right\} \subseteq L^{X}$.

We close this section by recalling the following definition of $L M$-fuzzy topology presented by [23].

Definition 5. [23] An $L M$-fuzzy topology $\mathfrak{T}$ on universe $X$ is a mapping $\mathfrak{T}$ : $L^{X} \rightarrow M$, such that for each $\lambda, \mu \in L^{X},\left\{\lambda_{i} \mid i \in I\right\} \subseteq L^{X}$ it satisfies,

(i) $\mathfrak{T}(\phi)=\mathfrak{T}(X)=1$,

(ii) $\mathfrak{T}(\lambda \wedge \mu) \geq \mathfrak{T}(\lambda) \wedge \mathfrak{T}(\mu)$,

(iii) $\mathfrak{T}\left(\vee_{i \in I} \lambda_{i}\right) \geq \wedge_{i \in I} \mathfrak{T}\left(\lambda_{i}\right)$.

For an LM-fuzzy topology $\mathfrak{T}$ on nonempty set $X$, the pair $(X, \mathfrak{T})$ is called an $L M$-fuzzy topological space. Further, $(X, \mathfrak{T})$ is

(vi) strong, if $\mathfrak{T}(\mathbf{a} \otimes \lambda) \geq \mathfrak{T}(\lambda)$,

(v) Alexandroff, if $\mathfrak{T}\left(\wedge_{i \in I} \lambda_{i}\right) \geq \wedge_{i \in I} \mathfrak{T}\left(\lambda_{i}\right)$.

Given two $L M$-fuzzy topological space $\left(X, \mathfrak{T}_{X}\right)$ and $\left(Y, \mathfrak{T}_{Y}\right)$ a map $f$ : $\left(X, \mathfrak{T}_{X}\right) \rightarrow\left(Y, \mathfrak{T}_{Y}\right)$ is called continuous if for all $\lambda \in L^{Y}, \mathfrak{T}_{X}\left(f^{\leftarrow}(\lambda)\right) \geq \mathfrak{T}_{Y}(\lambda)$. We denote by LM-ToP, the category of Alexandroff $L M$-fuzzy topological spaces.

The notion of $L M$-fuzzy co-topology [23] $\mathscr{T}$ can be defined similarly. Given two $L M$-fuzzy co-topological space $\left(X, \mathscr{T}_{X}\right)$ and $\left(Y, \mathscr{T}_{Y}\right)$ a map $f:\left(X, \mathscr{T}_{X}\right) \rightarrow$ $\left(Y, \mathscr{T}_{Y}\right)$ is called continuous if $\lambda \in L^{Y}, \mathcal{T}_{X}\left(f^{\leftarrow}(\lambda)\right) \geq \mathcal{T}_{Y}(\lambda)$. We denote by LM-CToP, the category of Alexandroff $L M$-fuzzy co-topological spaces.

\section{$3 \quad M$-valued Partition and Direct $F$-transforms}

In this section, we recall the notion of $M$-valued partitions and lattice-valued $F$-transforms [19]. We also discuss its behaviour based on ordering in spaces with $M$-valued partition. We begin with the following definition of $M$-valued partition as introduced in [19].

Definition 6. Let $X$ be a nonempty set. A collection $\mathcal{P}$ of normal $M$-valued sets $\left\{A_{\xi}: \xi \in \Lambda\right\}$ in $X$ is an $M$-valued partition of $X$, if $\left\{\operatorname{core}\left(A_{\xi}\right): \xi \in \Lambda\right\}$ is a partition (crisp) of $X . A$ pair $(X, \mathcal{P})$, where $\mathcal{P}$ is an $M$-valued partition of $X$, is called $a$ space with an $M$-valued partition.

Let $\mathcal{P}=\left\{A_{\xi}: \xi \in \Lambda\right\}$ be an $M$-valued partition of $X$. With this partition, we associate the following surjective index-function $i_{\mathcal{P}}: X \rightarrow \Lambda$ :

$$
i_{\mathcal{P}}(x)=\xi \Longleftrightarrow x \in \operatorname{core}\left(A_{\xi}\right) .
$$

Then $M$-valued partition $\mathcal{P}$ can be uniquely represented by the reflexive $M$-fuzzy relation $\delta_{\mathcal{P}}$ on $X$, such that

$$
\delta_{\mathcal{P}}(x, y)=A_{i_{\mathcal{P}}(x)}(y) .
$$


In [19], it has been proved that the relation (2) can be decomposed into a constituent $M$-fuzzy preorder relation $\delta_{\mathcal{P}_{\xi}}(x, y)$, where for each $\xi \in \Lambda$

$$
\delta_{\mathcal{P}_{\xi}}(x, y)= \begin{cases}A_{i_{\mathcal{P}}(x)}(y), & \text { if } x \in \operatorname{core}\left(A_{i_{\mathcal{P}}(x)}\right) \\ 1, & \text { if } x=y \\ 0, & \text { otherwise. }\end{cases}
$$

For given two spaces with $M$-valued partitions, following is the notion of morphism between them.

Definition 7. [15] Let $\left(X, \mathcal{P}=\left\{A_{\xi}: \xi \in \Lambda\right)\right\}$ and $\left(Y, \mathcal{Q}=\left\{B_{\omega}: \omega \in \Omega\right)\right\}$ be two spaces with $M$-valued partitions. $A$ morphism in the space with $M$-valued partitions is a pair of maps $(f, g)$, where $f: X \rightarrow Y$ and $g: \Lambda \rightarrow \Omega$ are maps such that for each $x \in X, \forall \xi \in \Lambda, \quad A_{\xi}(x) \leq B_{g(\xi)}(f(x))$.

It can be easily verified that all spaces with $M$-valued partition as objects and the pair of maps $(f, g)$ defined above as morphisms form a category [15], denoted by SMFP. If there is no danger of misunderstanding, the object-class of SMFP will be denoted by SMFP as well.

Let $L, M$ be iccl-monoids and $\psi: M \rightarrow L$ be the fixed mapping. Here, we define the following concept of lattice-valued direct $F^{\uparrow}\left(F^{\downarrow}\right)$-transforms.

Definition 8. Let $X$ be a nonempty set and $\mathcal{P}=\left\{A_{\xi}: \xi \in \Lambda\right\}$ be $M$-valued partition of $X$. Then for all $\lambda \in L^{X}$, and for all $x \in X$,

(i) direct $F^{\uparrow}$-transform of $L$-valued function $\lambda$ is defined by

$$
F_{\xi}^{\uparrow}(\lambda)=\vee_{y \in X}\left(\psi\left(A_{\xi}(y)\right) \otimes \lambda(y)\right)
$$

(ii) direct $F^{\downarrow}$-transform of L-valued function $\lambda$ is defined by

$$
F_{\xi}^{\downarrow}(\lambda)=\wedge_{y \in X}\left(\psi\left(A_{\xi}(y)\right) \rightarrow \lambda(y)\right) .
$$

It has been shown in [19] that for $M$-valued partition $\mathcal{P}=\left\{A_{\xi}: \xi \in \Lambda\right\}$ represented by $M$-fuzzy preorder relation $\delta_{\mathcal{P}_{\xi}}$ and with the associated index-function $i_{\mathcal{P}}$, we can associate two operators $F_{\mathcal{P}_{\xi}}^{\uparrow}$ and $F_{\mathcal{P}_{\xi}}^{\downarrow}$ corresponding to $\operatorname{direct} F^{\uparrow}$ and $F^{\downarrow}$-transforms. Where, for each $\lambda \in L^{X}$,

$$
\begin{aligned}
& F_{\mathcal{P}_{\xi}}^{\uparrow}(\lambda)(x)=F_{i_{\mathcal{P}}(x)}^{\uparrow}(\lambda)=\vee_{y \in X}\left(\psi\left(\delta_{\mathcal{P}_{\xi}}(x, y)\right) \otimes \lambda(y)\right), \\
& F_{\mathcal{P}_{\xi}}^{\downarrow}(\lambda)(x)=F_{i_{\mathcal{P}}(x)}^{\downarrow}(\lambda)=\wedge_{y \in X}\left(\psi\left(\delta_{\mathcal{P}_{\xi}}^{T}(x, y)\right) \rightarrow \lambda(y)\right) .
\end{aligned}
$$

In [19], it has been proved that the operators defined in Eqs. 3 and 4 connect the $i_{\mathcal{P}}(x)$-th upper (lower) $F^{\uparrow}\left(F^{\downarrow}\right)$-transform approximation with a closure (interior) operators in corresponding $L$-fuzzy co-topology (topology). 
Remark 1. Initially, the notion of lattice-valued direct $F$-transforms were introduced in [17], where the fuzzy partition $A_{\xi}$ was considered as a fuzzy subset of $[0,1]$ (i.e., $\left.A_{\xi}: X \rightarrow[0,1]\right)$ fulfilling the covering property, $(\forall x)(\exists \xi), A_{\xi}(x)>0$. Later, this notion were extended to the case where they are applied to $L$-valued functions in a space defined by $L$-valued fuzzy partitions, where $L$ is a complete residuated lattice. Here, if we consider the case where $L=M$ and $\psi=i d$ is an identity map, then the above definition of lattice-valued direct $F$-transforms will be similar to the lattice-valued direct $F$-transforms appeared in [17-20].

The following properties of the operators $F_{\mathcal{P}_{\xi}}^{\uparrow}$ and $F_{\mathcal{P}_{\xi}}^{\downarrow}$ were presented in $[17,19]$. All of them are formulated below for arbitrary $\mathbf{a}, \lambda, \lambda_{i} \in L^{X}$.

Proposition 3. Let $X$ be a nonempty set and $\mathcal{P}$ be the $M$-valued partition of $X$. Then for all $\mathbf{a}, \lambda \in L^{X}$, and $\left\{\lambda_{i} \mid i \in I\right\} \subseteq L^{X}$, the operators $F_{\mathcal{P}_{\xi}}^{\uparrow}: L^{X} \rightarrow L^{X}$ and $F_{\mathcal{P}_{\xi}}^{\downarrow}: L^{X} \rightarrow L^{X}$ satisfies the following properties.

(i) $F_{\mathcal{P}_{\xi}}^{\uparrow}(\mathbf{a})=\mathbf{a}, \quad F_{\mathcal{P}_{\xi}}^{\downarrow}(\mathbf{a})=\mathbf{a}$,

(ii) $\lambda \leq F_{\mathcal{P}_{\xi}}^{\uparrow}(\lambda), \quad F_{\mathcal{P}_{\xi}}^{\downarrow}(\lambda) \leq \lambda$,

(iii) $F_{\mathcal{P}_{\xi}}^{\uparrow}\left(\vee_{i \in I} \lambda_{i}\right)=\vee_{i \in I} F_{\mathcal{P}_{\xi}}^{\uparrow}\left(\lambda_{i}\right), F_{\mathcal{P}_{\xi}}^{\downarrow}\left(\wedge_{i \in I} \lambda_{i}\right)=\wedge_{i \in I} F_{\mathcal{P}_{\xi}}^{\downarrow}\left(\lambda_{i}\right)$,

(iv) $F_{\mathcal{P}_{\xi}}^{\uparrow}(\mathbf{a} \otimes \lambda)=a \otimes F_{\mathcal{P}_{\xi}}^{\uparrow}(\lambda), \quad F_{\mathcal{P}_{\xi}}^{\downarrow}(\mathbf{a} \rightarrow \lambda)=a \rightarrow F_{\mathcal{P}_{\xi}}^{\downarrow}(\lambda)$.

Below, we investigate the properties of lattice-valued direct $F$-transforms with respect to ordering between spaces with $M$-valued partition. We have the following.

Proposition 4. Let $(f, g):(X, \mathcal{P}) \rightarrow(Y, \mathcal{Q})$ be a morphism between spaces with $M$-valued partition and fixed mapping $\psi$ is monotonic decreasing. Then,

(i) $F_{\xi}^{\uparrow}\left(f^{\leftarrow}(\lambda)\right) \leq F_{g(\xi)}^{\uparrow}(\lambda)$,

(ii) $F_{\xi}^{\downarrow}\left(f^{\leftarrow}(\lambda)\right) \geq F_{g(\xi)}^{\downarrow}(\lambda)$,

(iii) $F_{g(\xi)}^{\uparrow}(f \rightarrow(\lambda)) \geq F_{\xi}^{\uparrow}(\lambda)$,

(iv) $F_{g(\xi)}^{\downarrow}(f \rightarrow(\lambda)) \leq F_{\xi}^{\downarrow}(\lambda)$.

Proof. (i) Let $\lambda \in L^{Y}$. From Definition 8, we have

$$
\begin{aligned}
F_{\xi}^{\uparrow}\left(f^{\leftarrow}(\lambda)\right) & =\vee_{x \in X} \psi\left(A_{\xi}(x)\right) \otimes f^{\leftarrow}(\lambda)(x)=\vee_{x \in X} \psi\left(A_{\xi}(x)\right) \otimes \lambda(f(x)) \\
& \leq \vee_{x \in X} \psi\left(B_{g(\xi)}(f(x))\right) \otimes \lambda(f(x)) \leq \vee_{y \in Y} \psi\left(B_{g(\xi)}(y)\right) \otimes \lambda(y) \\
& =F_{g(\xi)}^{\uparrow}(\lambda) .
\end{aligned}
$$

(ii) Let $\lambda \in L^{Y}$. From Definition 8, we get

$$
\begin{aligned}
F_{\xi}^{\downarrow}\left(f^{\leftarrow}(\lambda)\right) & =\wedge_{x \in X} \psi\left(A_{\xi}(x)\right) \rightarrow f^{\leftarrow}(\lambda)(x)=\wedge_{x \in X} \psi\left(A_{\xi}(x)\right) \rightarrow \lambda(f(x)) \\
& \geq \wedge_{x \in X} \psi\left(B_{g(\xi)}(f(x))\right) \rightarrow \lambda(f(x)) \geq \wedge_{y \in Y} \psi\left(B_{g(\xi)}(y)\right) \rightarrow \lambda(y) \\
& =F_{g(\xi)}^{\downarrow}(\lambda) .
\end{aligned}
$$


(iii) Let $\lambda \in L^{X}$. From Definition 8, we obtain $F_{g(\xi)}^{\uparrow}(f \rightarrow(\lambda))$

$$
\begin{aligned}
& =\vee_{y \in Y}\left(f^{\rightarrow}(\lambda)(y) \otimes \psi\left(B_{g(\xi)}(y)\right)\right)=\vee_{y \in Y}\left(\vee_{x: f(x)=y} \lambda(x) \otimes \psi\left(B_{g(\xi)}(y)\right)\right) \\
& \geq \vee_{x \in X} \lambda(x) \otimes \psi\left(B_{g(\xi)}(f(x))\right) \geq \vee_{x \in X} \lambda(x) \otimes \psi\left(A_{\xi}(x)\right)=F_{\xi}^{\uparrow}(\lambda) .
\end{aligned}
$$

(iv) Let $\lambda \in L^{X}$. From Definition 8, we obtain

$$
\begin{aligned}
& F_{g(\xi)}^{\downarrow}(f \rightarrow(\lambda)) \\
= & \wedge_{y \in Y}\left(\psi\left(B_{g(\xi)}(y)\right) \rightarrow f^{\rightarrow}(\lambda)(y)\right)=\wedge_{y \in Y}\left(\psi\left(B_{g(\xi)}(y)\right) \rightarrow \vee_{x: f(x)=y} \lambda(x)\right) \\
= & \wedge_{x \in X} \psi\left(B_{g(\xi)}(y)(f(x)) \rightarrow \lambda(x) \leq \wedge_{x \in X} \psi\left(A_{\xi}(x)\right) \rightarrow \lambda(x)=F_{\xi}^{\downarrow}(\lambda) .\right.
\end{aligned}
$$

\section{Main Results}

In this section, we introduce and study the measure of lattice-valued direct $F$-transforms of an $L$-fuzzy set. The defined measure determines the amount of preciseness of $L$-fuzzy subset into $L$-fuzzy set. Further, we investigate the topologies induced by the measure of lattice-valued direct $F$-transform operators. In particular, we show that every measure of lattice-valued direct $F$-transform operators determine strong Alexandroff $L M$-fuzzy topological and co-topological spaces.

Definition 9. Let $X$ be a nonempty set and $\mathcal{P}$ be the $M$-valued partition of $X$. Then for an L-fuzzy set $\lambda \in L^{X}$. The $M$-valued measure of direct $F^{\uparrow}$ transform $\mathcal{F}_{u}(\lambda)=F_{\mathcal{P}_{\xi}}^{\uparrow}(\lambda) \hookrightarrow \lambda$ and $M$-valued measure of direct $F^{\downarrow}$-transform $\mathcal{F}_{l}(\lambda)=\lambda \hookrightarrow F_{\mathcal{P}_{\xi}}^{\downarrow}(\lambda)$ are maps $\mathcal{F}_{u}, \mathcal{F}_{l}: L^{X} \rightarrow M$ and defined as,

$$
\mathcal{F}_{u}(\lambda)=\phi\left(\wedge_{x \in X}\left(F_{\mathcal{P}_{\xi}}^{\uparrow}(\lambda)(x) \rightarrow \lambda(x)\right)\right), \quad \mathcal{F}_{l}(\lambda)=\phi\left(\wedge_{x \in X}\left(\lambda(x) \rightarrow F_{\mathcal{P}_{\xi}}^{\downarrow}(\lambda)(x)\right)\right) .
$$

To investigate in more detail how the $M$-valued measure of $F$-transform operators preserve the inclusion of two sets defined in the real interval $[0,1]$, instead of a general complete residuated lattice $L$, we will use the specific example of residuated lattices.

Example 1. Consider the case $L=M$, and $\phi=\psi=i d$ and the Lukasiewicz algebra

$$
\mathscr{L}_{L}=([0,1], \vee, \wedge, \otimes, \rightarrow, 0,1)
$$

Then the measure of lattice-valued $F$-transform operators of an $L$-fuzzy set is obtained as below,

$$
\begin{aligned}
\mathcal{F}_{u} & =\wedge_{x, y \in X}\left(2-\lambda(x)+\lambda(y)-A_{i_{\mathcal{P}}(y)}(x)\right), \\
\mathcal{F}_{l} & =\wedge_{x, y \in X}\left(2-\lambda(x)+\lambda(y)-A_{i_{\mathcal{P}}(x)}(y)\right) .
\end{aligned}
$$


Proposition 5. Let $X$ be a nonempty set and $\mathcal{P}$ be the $M$-valued partition of $X$. Then for each $\mathbf{a}, \lambda \in L^{X}$ and for all $\left\{\lambda_{i} \mid i \in I\right\} \subseteq L^{X}$, the $M$-valued measure of F-transform operators $\mathcal{F}_{u}, \mathcal{F}_{l}: L^{X} \rightarrow M$ satisfy the following properties.

(i) $\mathcal{F}_{u}(\mathbf{a})=1_{M}, \mathcal{F}_{l}(\mathbf{a})=1_{M}$,

(ii) $\mathcal{F}_{u}\left(\vee_{i \in I} \lambda_{i}\right) \geq \wedge_{i \in I} \mathcal{F}_{u}\left(\lambda_{i}\right), \mathcal{F}_{l}\left(\vee_{i \in I} \lambda_{i}\right) \geq \wedge_{i \in I} \mathcal{F}_{l}\left(\lambda_{i}\right)$,

(iii) $\mathcal{F}_{u}\left(\wedge_{i \in I} \lambda_{i}\right) \geq \wedge_{i \in I} \mathcal{F}_{u}\left(\lambda_{i}\right), \mathcal{F}_{l}\left(\wedge_{i \in I} \lambda_{i}\right) \geq \wedge_{i \in I} \mathcal{F}_{l}\left(\lambda_{i}\right)$,

(iv) $\mathcal{F}_{u}(\mathbf{a} \otimes \lambda) \geq \mathcal{F}_{u}(\lambda), \mathcal{F}_{l}(\mathbf{a} \rightarrow \lambda) \geq \mathcal{F}_{l}(\lambda)$.

Proof. We give only proof for the $M$-valued measure of direct $F$ transform operator $\mathcal{F}_{u}$. The proof for operator $\mathcal{F}_{l}$ follows similarly.

(i) For all $\mathbf{a} \in L^{X}$, and from Proposition 3, we have,

$$
\begin{aligned}
\mathcal{F}_{u}(\mathbf{a})=F_{\mathcal{P}_{\xi}}^{\uparrow}(\mathbf{a}) \hookrightarrow \mathbf{a} & =\phi\left(\wedge_{x \in X}\left(F_{\mathcal{P}_{\xi}}^{\uparrow}(\mathbf{a})(x) \rightarrow \mathbf{a}(x)\right)\right) \\
& =\phi\left(\wedge_{x \in X}(\mathbf{a}(x) \rightarrow \mathbf{a}(x))\right)=1_{M}
\end{aligned}
$$

(ii) For all $\lambda \in L^{X}$, and $\left\{\lambda_{i} \mid i \in I\right\} \subseteq L^{X}$, from Proposition 2, we have

$$
\begin{aligned}
\mathcal{F}_{u}\left(\bigvee_{i \in I} \lambda_{i}\right) & =F_{\mathcal{P}_{\xi}}^{\uparrow}\left(\vee_{i \in I} \lambda_{i}\right) \hookrightarrow\left(\bigvee_{i \in I} \lambda_{i}\right) \\
& \left.=\vee_{i \in I}\left(F_{\mathcal{P}_{\xi}}^{\uparrow}\left(\lambda_{i}\right)\right) \hookrightarrow\left(\bigvee_{i \in I} \lambda_{i}\right) \geq \wedge_{i \in I}\left(F_{\mathcal{P}_{\xi}}^{\uparrow}\left(\lambda_{i}\right)\right) \hookrightarrow\left(\lambda_{i}\right)\right) \\
& =\wedge_{i \in I} \mathcal{F}_{u}\left(\lambda_{i}\right) .
\end{aligned}
$$

(iii) For all $\lambda \in L^{X}$, and $\left\{\lambda_{i} \mid i \in I\right\} \subseteq L^{X}$, from Proposition 2, we have

$$
\begin{aligned}
\mathcal{F}_{u}\left(\wedge_{i \in I} \lambda_{i}\right) & =F_{\mathcal{P}_{\xi}}^{\uparrow}\left(\wedge_{i \in I} \lambda_{i}\right) \hookrightarrow\left(\wedge_{i \in I} \lambda_{i}\right) \\
& \left.\geq \wedge_{i \in I}\left(F_{\mathcal{P}_{\xi}}^{\uparrow}\left(\lambda_{i}\right)\right) \hookrightarrow\left(\wedge_{i \in I} \lambda_{i}\right) \geq \wedge_{i \in I}\left(F_{\mathcal{P}_{\xi}}^{\uparrow}\left(\lambda_{i}\right)\right) \hookrightarrow\left(\lambda_{i}\right)\right) \\
& =\wedge_{i \in I} \mathcal{F}_{u}\left(\lambda_{i}\right) .
\end{aligned}
$$

(iv) For all $\mathbf{a}, \lambda \in L^{X}$, from Proposition 3, we have,

$$
\begin{aligned}
\mathcal{F}_{u}(\mathbf{a} \otimes \lambda) & =F_{\mathcal{P}_{\xi}}^{\uparrow}(\mathbf{a} \otimes \lambda) \hookrightarrow(\mathbf{a} \otimes \lambda) \\
& =\phi\left(\wedge_{x \in X}\left(\vee_{y \in X}\left(\psi\left(A_{i_{\mathcal{P}}(x)}(y)\right) \otimes(\mathbf{a} \otimes \lambda)(y)\right) \rightarrow(\mathbf{a} \otimes \lambda)(x)\right)\right) \\
& \geq \phi\left(\wedge_{x \in X}\left(\vee_{y \in X}\left(\psi\left(A_{i_{\mathcal{P}}(x)}(y)\right) \otimes \lambda(y)\right) \rightarrow \lambda(x)\right)\right) \\
& =\phi\left(\wedge_{x \in X}\left(F_{\mathcal{P}_{\xi}}^{\uparrow}(\lambda)(x) \rightarrow \lambda(x)\right)\right)=\mathcal{F}_{u}(\lambda) .
\end{aligned}
$$

\subsection{Measure of Direct $F^{\downarrow}$-transform Operator and $L M$-fuzzy Topology}

In this subsection, we show that the $M$-valued measure of direct $F^{\downarrow}$-transform induces $L M$-fuzzy topology. 
Theorem 1. Let $(X, \mathcal{P})$ be a space with an $M$-valued partition, $\delta_{\mathcal{P}_{\xi}}$ representing $M$-fuzzy preorder relation and $F_{\mathcal{P}_{\xi}}^{\downarrow}: L^{X} \rightarrow L^{X}$ be the corresponding $F^{\downarrow}$-operator. Then the pair $\left(X, \mathfrak{T}_{\mathcal{P}}\right)$, where $\mathfrak{T}_{\mathcal{P}}=\left\{\mathcal{F}_{l}(\cdot)(x): L^{X} \rightarrow M \mid x \in X\right\}$ is such that for every $\lambda \in L^{X}$ and $x \in X$,

$$
\mathcal{F}_{l}(\lambda)=\lambda \hookrightarrow F_{\mathcal{P}_{\xi}}^{\downarrow}(\lambda)
$$

is a strong Alexandroff LM-fuzzy topological space.

Proof. Let $\mathcal{P}=\left\{A_{\xi}: \xi \in \Lambda\right\}$ be an $M$-valued partition and $i_{\mathcal{P}}$ be the corresponding index-function, such that for all $x \in X$, the value $i_{\mathcal{P}}(x)$ determines the unique partition element $A_{i_{\mathcal{P}}(x)}$, where $x \in \operatorname{core}\left(A_{i_{\mathcal{P}}(x)}\right)$. For every $x \in X, \lambda \in L^{X}$, we claim that $F_{\mathcal{P}_{\xi}}^{\downarrow}(\lambda)(x)=F_{i_{\mathcal{P}}(x)}^{\downarrow}(\lambda)$, where the right-hand side is the $i_{\mathcal{P}}(x)$-th $F^{\downarrow}$ transform component of $\lambda$. Indeed, for a particular $x \in X, F_{i_{\mathcal{P}}(x)}^{\downarrow}(\lambda)$ is computed in accordance with (4) and by this, coincides with $F_{\mathcal{P}_{\xi}}^{\downarrow}(\lambda)(x)$. Now it only remains to verify that the collection $\mathfrak{T}_{\mathcal{P}}=\left\{\mathcal{F}_{l}(\cdot)(x): L^{X} \rightarrow M \mid x \in X\right\}$, where for every $\lambda \in L^{X}, \mathcal{F}_{l}(\lambda)=\lambda \hookrightarrow F_{i_{\mathcal{P}}(x)}^{\downarrow}(\lambda)$ is a strong Alexandroff $L M$-fuzzy topological space. It can be seen that the properties (i)-(iv) of Proposition 5 characterize the $M$-valued measure of direct $F^{\downarrow}$-transform operator $\mathcal{F}_{l}: L^{X} \rightarrow M$ as strong Alexandroff $L M$-fuzzy topological space.

Theorem 2. Let $(f, g):(X, \mathcal{P}) \rightarrow(Y, \mathcal{Q})$ be a morphism between the spaces with $M$-valued partition, characterized by index-functions $i_{\mathcal{P}}$ and $k_{\mathcal{P}}$, respectively. Let, moreover, Alexandroff LM-fuzzy topologies $\mathfrak{T}_{X}=\left\{\mathcal{F}_{l}^{i_{\mathcal{P}}}: L^{X} \rightarrow M \mid x \in X\right\}$ on $X$ and $\mathfrak{T}_{Y}=\left\{\mathcal{F}_{l}^{k_{\mathcal{P}}}: L^{Y} \rightarrow M \mid y \in Y\right\}$ on $Y$ be induced by the $F^{\downarrow}$-transform. Then $f:\left(X, \mathfrak{T}_{X}\right) \rightarrow\left(Y, \mathfrak{T}_{Y}\right)$ is a continuous map.

Proof. Since $(f, g):(X, \mathcal{P}) \rightarrow(Y, \mathcal{Q})$ be an FP-map. Then from Definition 7 , for all $\xi \in \Lambda, A_{\xi}(x) \leq B_{g(\xi)}(f(x))$. Now we have to show that for all $\lambda \in L^{Y}$, $\mathfrak{T}_{X}\left(f^{\leftarrow}(\lambda)\right) \geq \mathfrak{T}_{Y}(\lambda)$, where

$$
\begin{aligned}
\mathfrak{T}_{X}\left(f^{\leftarrow}(\lambda)\right) & =\mathcal{F}_{l}^{i_{\mathcal{P}}}\left(f^{\leftarrow}(\lambda)\right)=f^{\leftarrow}(\lambda) \hookrightarrow F_{i_{\mathcal{P}}(x)}^{\downarrow}\left(f^{\leftarrow}(\lambda)\right) \\
& =\phi\left(\wedge_{x \in X}\left(f^{\leftarrow}(\lambda)(x) \rightarrow F_{i_{\mathcal{P}}(x)}^{\downarrow}\left(f^{\leftarrow}(\lambda)(x)\right)\right)\right) \\
& =\phi\left(\wedge_{x \in X}\left(\lambda(f(x)) \rightarrow \wedge_{t \in X}\left(\psi\left(A_{i_{\mathcal{P}}(x)}(t)\right) \rightarrow \lambda(f(t))\right)\right)\right. \\
& =\phi\left(\wedge_{x \in X} \wedge_{t \in X}\left(\lambda(f(x)) \rightarrow\left(\psi\left(A_{i_{\mathcal{P}}(x)}(t)\right) \rightarrow \lambda(f(t))\right)\right)\right. \\
& \geq \phi\left(\wedge_{x \in X} \wedge_{t \in X}\left(\lambda(f(x)) \rightarrow\left(\psi\left(B_{k_{\mathcal{P}}(x)}(f(t))\right) \rightarrow \lambda(f(t))\right)\right)\right. \\
& \geq \phi\left(\wedge_{z \in X} \wedge_{y \in X}\left(\lambda(z) \rightarrow\left(\psi\left(B_{k_{\mathcal{P}}(x)}(y)\right) \rightarrow \lambda(y)\right)\right)\right. \\
& \geq \phi\left(\wedge_{z \in X}\left(\lambda(z) \rightarrow \wedge_{y \in X}\left(\psi\left(B_{k_{\mathcal{P}}(x)}(y)\right) \rightarrow \lambda(y)\right)\right)\right. \\
& =\lambda \hookrightarrow F_{k_{\mathcal{P}}(z)}^{\downarrow}(\lambda)=\mathcal{F}_{l}^{k \mathcal{P}}(\lambda)=\mathfrak{T}_{Y}(\lambda) .
\end{aligned}
$$

Thus $f:\left(X, \mathfrak{T}_{X}\right) \rightarrow\left(Y, \mathfrak{T}_{Y}\right)$ is a continuous map. 
From Theorems 1, 2, we obtain a functor $\mathbb{G}$ as follows:

$$
\mathbb{G}:\left\{\begin{aligned}
\mathbf{S M F P} & \longmapsto \mathbf{L M}-\mathbf{T o P} \\
(X, \mathcal{P}) & \longmapsto\left(X, \mathfrak{T}_{X}\right) \\
(f, g) & \longmapsto f,
\end{aligned}\right.
$$

where $\mathcal{P}=\left\{A_{\xi}: \xi \in \Lambda\right\}$ is an $M$-valued partition of $X, \mathfrak{T}_{X}=\left\{\mathcal{F}_{l}^{i_{\mathcal{P}}}: L^{X} \rightarrow M \mid\right.$ $x \in X\}$ is the induced strong Alexandroff $L M$-fuzzy topology on $X$ by the $M$ valued measure of direct $F^{\downarrow}$-transform operator $\mathcal{F}_{l}$, and $(f, g):(X, \mathcal{P}) \rightarrow(Y, \mathcal{Q})$ is a morphism between the spaces with $M$-valued partition.

\subsection{Measure of Direct $F^{\uparrow}$-transform Operator and $L M$-fuzzy Co-topology}

Here, we show that the $M$-valued measure of direct $F^{\uparrow}$-transform induces $L M$ fuzzy co-topology.

Theorem 3. Let $(X, \mathcal{P})$ be a space with an $M$-valued partition, $\delta_{\mathcal{P}_{\xi}}$ representing $M$-fuzzy preorder relation and $F_{\mathcal{P}_{\xi}}^{\uparrow}: L^{X} \rightarrow L^{X}$ be the corresponding $F^{\uparrow}$-operator. Then the pair $\left(X, \mathcal{T}_{\mathcal{P}}\right)$, where $\mathcal{T}_{\mathcal{P}}=\left\{\mathcal{F}_{u}(\cdot)(x): L^{X} \rightarrow M \mid x \in X\right\}$ is such that for every $\lambda \in L^{X}$ and $x \in X$,

$$
\mathcal{F}_{u}(\lambda)=F_{\mathcal{P}_{\xi}}^{\uparrow}(\lambda) \hookrightarrow \lambda
$$

is a strong Alexandroff LM-fuzzy co-topological space.

Proof. The proof is analogous to Theorem 1.

Theorem 4. Let $(f, g):(X, \mathcal{P}) \rightarrow(Y, \mathcal{Q})$ be a morphism between the spaces with $M$-valued partition, characterized by index-functions $i_{\mathcal{P}}$ and $k_{\mathcal{P}}$, respectively. Let, moreover, Alexandroff LM-fuzzy co-topologies $\mathcal{T}_{X}=\left\{\mathcal{F}_{u}^{i_{\mathcal{P}}}: L^{X} \rightarrow M \mid x \in X\right\}$ on $X$ and $\mathcal{T}_{Y}=\left\{\mathcal{F}_{u}^{k_{\mathcal{P}}}: L^{Y} \rightarrow M \mid y \in Y\right\}$ on $Y$ be induced by the $F^{\uparrow}$-transform. Then $f:\left(X, \mathcal{T}_{X}\right) \rightarrow\left(Y, \mathcal{T}_{Y}\right)$ is a continuous map.

Proof. Since $(f, g):(X, \mathcal{P}) \rightarrow(Y, \mathcal{Q})$ be a morphism between space with $M$ valued partitions. Then from Definition 7, for all $\xi \in \Lambda, A_{\xi}(x) \leq B_{g(\xi)}(f(x))$. Now we have to show that for all $\lambda \in L^{Y}, \mathcal{T}_{X}\left(f^{\leftarrow}(\lambda)\right) \geq \mathcal{T}_{Y}(\lambda)$, where

$$
\begin{aligned}
\mathcal{T}_{X}\left(f^{\leftarrow}(\lambda)\right) & =\mathcal{F}_{u}^{i_{\mathcal{P}}}\left(f^{\leftarrow}(\lambda)\right)=F_{i_{\mathcal{P}}(x)}^{\uparrow}\left(f^{\leftarrow}(\lambda)\right) \hookrightarrow f^{\leftarrow}(\lambda) \\
& =\phi\left(\wedge_{x \in X}\left(F_{i_{\mathcal{P}}(x)}^{\uparrow}\left(f^{\leftarrow}(\lambda)(x)\right) \rightarrow f^{\leftarrow}(\lambda)(x)\right)\right) \\
& =\phi\left(\wedge_{x \in X}\left(\wedge_{t \in X}\left(\psi\left(A_{i_{\mathcal{P}}(x)}(t)\right) \otimes \lambda(f(t))\right) \rightarrow \lambda(f(x))\right)\right. \\
& =\phi\left(\wedge_{x \in X} \wedge_{t \in X}\left(\psi\left(A_{i_{\mathcal{P}}(x)}(t)\right) \otimes \lambda(f(t))\right) \rightarrow \lambda(f(x))\right) \\
& \geq \phi\left(\wedge_{x \in X} \wedge_{t \in X}\left(\psi\left(B_{k_{\mathcal{P}}(x)}(f(t))\right) \otimes \lambda(f(t))\right) \rightarrow \lambda(f(x))\right) \\
& \geq \phi\left(\wedge_{z \in X} \wedge_{y \in X}\left(\psi\left(B_{k_{\mathcal{P}}(x)}(y)\right) \otimes \lambda(y)\right) \rightarrow \lambda(z)\right) \\
& \geq \phi\left(\wedge_{z \in X} \wedge_{y \in X}\left(\psi\left(B_{k_{\mathcal{P}}(x)}(y)\right) \otimes \lambda(y)\right) \rightarrow(\lambda(z))\right. \\
& =F_{k_{\mathcal{P}}(z)}^{\uparrow}(\lambda) \hookrightarrow \lambda=\mathcal{F}_{u}^{k_{\mathcal{P}}}(\lambda)=\mathcal{T}_{Y}(\lambda) .
\end{aligned}
$$


Thus $f:\left(X, \mathcal{T}_{X}\right) \rightarrow\left(Y, \mathcal{T}_{Y}\right)$ is a continuous map.

From Theorems 3, 4, we obtain a functor $\mathbb{H}$ as follows:

$$
\mathbb{H}:\left\{\begin{aligned}
\text { SMFP } & \longmapsto \text { LM-CToP } \\
(X, \mathcal{P}) & \longmapsto\left(X, \mathcal{T}_{X}\right) \\
(f, g) & \longmapsto f
\end{aligned}\right.
$$

where $\mathcal{P}=\left\{A_{\xi}: \xi \in \Lambda\right\}$ is an $M$-valued partition of $X, \mathcal{T}_{X}=\left\{\mathcal{F}_{u}^{i_{\mathcal{P}}}: L^{X} \rightarrow M\right.$ $x \in X\}$ is the induced strong Alexandroff $L M$-fuzzy co-topology on $X$ by the measure of direct $F^{\uparrow}$-transform operator $\mathcal{F}_{u}$, and $(f, g):(X, \mathcal{P}) \rightarrow(Y, \mathcal{Q})$ is a morphism between the spaces with $M$-valued partition.

Below, we show that the $M$-valued measure of $F$-transform operators $\mathcal{F}_{u}$, $\mathcal{F}_{l}$ together with its induced $L M$-fuzzy topologies $\mathfrak{T}, \mathcal{T}$ can be interpreted as $L M$-fuzzy ditopology.

Corollary 1. Let $(X, \mathcal{P})$ be space with $M$-valued partition, $\mathfrak{T}_{\mathcal{P}}=\left\{\mathcal{F}_{l}(\cdot)(x)\right.$ : $\left.L^{X} \rightarrow M \mid x \in X\right\}$ and $\mathcal{T}_{\mathcal{P}}=\left\{\mathcal{F}_{u}(\cdot)(x): L^{X} \rightarrow M \mid x \in X\right\}$ be the induced strong Alexandroff LM-fuzzy topology and co-topology. Then the triple $\left(X, \mathfrak{T}_{\mathcal{P}_{X}}, \mathcal{T}_{\mathcal{P}_{X}}\right)$ is a strong Alexandroff $L M$-fuzzy ditopology.

The following proposition is an easy consequence of Theorems 2 and 4.

Proposition 6. Let $(f, g):(X, \mathcal{P}) \rightarrow(Y, \mathcal{Q})$ be a morphism between the spaces with $M$-valued partition. Let, moreover, assume that the assumptions of Theorems 2 and 4 holds. Then $f:\left(X, \mathfrak{T}_{\mathcal{P}_{X}}, \mathcal{T}_{\mathcal{P}_{X}}\right) \rightarrow\left(Y, \mathfrak{T}_{\mathcal{P}_{Y}}, \mathcal{T}_{\mathcal{P}_{Y}}\right)$ is a continuous mapping between two strong Alexandroff LM-fuzzy ditopological spaces.

From the above two results we obtain a functor $\mathbb{L}$ as follows:

$$
\mathbb{L}:\left\{\begin{aligned}
\text { SMFP } & \longmapsto \text { LM-DIToP } \\
(X, \mathcal{P}) & \longmapsto\left(X, \mathfrak{T}_{\mathcal{P}_{X}}, \mathcal{T}_{\mathcal{P}_{X}}\right) \\
(f, g) & \longmapsto f .
\end{aligned}\right.
$$

\section{Conclusion}

The paper is an effort to show that by defining the $M$-valued measure of $F$ transform operators, we can associate Alexandroff $L M$-fuzzy topological and co-topological spaces. Specifically, we have introduced and studied the $M$-valued measure of inclusion defined between the lattice-valued $F^{\uparrow}$ and $F^{\downarrow}$-transform and $L$-fuzzy set. The basic properties of defined operators are studied. The $M$-valued measure of $F$-transforms defined here are essentially in some sense determine the amount of preciseness of given $L$-fuzzy set. We have discussed such connections through the categorical point of view. 
We believe that the $M$-valued measure of $F$-transform operators propose an abstract approach to the notion of "precision of approximation" and naturally arise in connection with applications to image and data analysis etc. Which will be one of our directions for the future work. In another direction, we propose to investigate the categorical behavior of the operators $\mathcal{F}_{u}, \mathcal{F}_{l}$ in a more deeper way.

Acknowledgment. The work of first author is supported by University of Ostrava grant IRP201824 "Complex topological structures" and the work of Irina Perfilieva is partially supported by the Grant Agency of the Czech Republic (project No. 1806915S).

\section{References}

1. Blount, K., Tsinakis, C.: The structure of residuated lattices. Int. J. Algebr. Comput. 13(04), 437-461 (2003)

2. Bustince, H.: Indicator of inclusion grade for interval-valued fuzzy sets. Application to approximate reasoning based on interval-valued fuzzy sets. Int. J. Approximate Reasoning 23(3), 137-209 (2000)

3. Di Martino, F., Loia, V., Perfilieva, I., Sessa, S.: An image coding/decoding method based on direct and inverse fuzzy transforms. Int. J. Approximate Reasoning 48(1), 110-131 (2008)

4. Goguen, J.A.: L-fuzzy sets. J. Math. Anal. Appl. 18, 145-174 (1967)

5. Hájek, P.: Metamathematics of Fuzzy Logic, vol. 4. Springer, Dordrecht (2013). https://doi.org/10.1007/978-94-011-5300-3

6. Han, S.-E., Šstak, A.: M-valued measure of roughness for approximation of $L$ fuzzy sets and its topological interpretation. In: Merelo, J.J., Rosa, A., Cadenas, J.M., Dourado, A., Madani, K., Filipe, J. (eds.) Computational Intelligence. SCI, vol. 620, pp. 251-266. Springer, Cham (2016). https://doi.org/10.1007/978-3-31926393-9_15

7. Han, S.E., Šostak, A.: On the measure of M-rough approximation of L-fuzzy sets. Soft Comput. 22(12), 3843-3855 (2018). https://doi.org/10.1007/s00500-0172841-y

8. Höhle, U.: M-valued sets and sheaves over integral commutative CL-monoids. In: Rodabaugh, S.E., Klement, E.P., Höhle, U. (eds.) Applications of Category Theory to Fuzzy Subsets. Theory and Decision Library (Series B: Mathematical and Statistical Methods), vol. 14, pp. 33-72. Springer, Dordrecht (1992). https://doi. org/10.1007/978-94-011-2616-8_3

9. Höhle, U.: Commutative, residuated L-monoids. In: Höhle, U., Klement, E.P. (eds.) Non-Classical Logics and their Applications to Fuzzy Subsets. Theory and Decision Library (Series B: Mathematical and Statistical Methods), vol. 32 pp. 53-106. Springer, Dordrecht (1995). https://doi.org/10.1007/978-94-011-0215-5_5

10. Järvinen, J.: On the structure of rough approximations. Fundam. Inform. 53(2), 135-153 (2002)

11. Järvinen, J., Kortelainen, J.: A unifying study between modal-like operators, topologies and fuzzy sets. Fuzzy Sets Syst. 158(11), 1217-1225 (2007)

12. Kehagias, A., Konstantinidou, M.: L-fuzzy valued inclusion measure, l-fuzzy similarity and l-fuzzy distance. Fuzzy Sets Syst. 136(3), 313-332 (2003) 
13. Khastan, A., Perfilieva, I., Alijani, Z.: A new fuzzy approximation method to cauchy problems by fuzzy transform. Fuzzy Sets Syst. 288, 75-95 (2016)

14. Močkoř, J.: Axiomatic of lattice-valued F-transform. Fuzzy Sets Syst. 342, 53-66 (2018)

15. Močkoř, J., Holčapek, M.: Fuzzy objects in spaces with fuzzy partitions. Soft Comput. 21, 7269-7284 (2017). https://doi.org/10.1007/s00500-016-2431-4

16. Močkor, J., Hurtik, P.: Lattice-valued F-transforms and similarity relations. Fuzzy Sets Syst. 342, 67-89 (2018)

17. Perfilieva, I.: Fuzzy transforms: theory and applications. Fuzzy Sets Syst. 157(8), 993-1023 (2006)

18. Perfilieva, I., Singh, A.P., Tiwari, S.P.: On F-transforms, L-fuzzy partitions and L-fuzzy pretopological spaces. In: 2017 IEEE Symposium Series on Computational Intelligence (SSCI), pp. 1-8. IEEE (2017)

19. Perfilieva, I., Singh, A.P., Tiwari, S.P.: On the relationship among F-transform, fuzzy rough set and fuzzy topology. Soft Comput. 21, 3513-3523 (2017). https:// doi.org/10.1007/s00500-017-2559-x

20. Perfilieva, I., Tiwari, S.P., Singh, A.P.: Lattice-valued F-transforms as interior operators of L-fuzzy pretopological spaces. In: Medina, J., et al. (eds.) IPMU 2018. CCIS, vol. 854, pp. 163-174. Springer, Cham (2018). https://doi.org/10.1007/9783-319-91476-3_14

21. Qin, K., Pei, Z.: On the topological properties of fuzzy rough sets. Fuzzy Sets Syst. 151, 601-613 (2005)

22. Singh, A.P., Tiwari, S.P., Perfilieva, I.: F-transforms, L-fuzzy partitions and L-fuzzy pretopological spaces: an operator oriented view. Fuzzy Sets Syst. Submitted

23. Sostak, A., Brown, L.M.: Categories of fuzzy topology in the context of graded ditopologies on textures. Iran. J. Fuzzy Syst. 11(6), 1-20 (2014)

24. Stepnicka, M., Valasek, R.: Numerical solution of partial differential equations with help of fuzzy transform. In: The 14th IEEE International Conference on Fuzzy Systems. FUZZ 2005, pp. 1104-1109. IEEE (2005)

25. Tiwari, S.P., Perfilieva, I., Singh, A.P.: Generalized residuated lattices based Ftransform. Iran. J. Fuzzy Syst. 15(2), 165-182 (2018)

26. Tiwari, S.P., Srivastava, A.K.: Fuzzy rough sets, fuzzy preorders and fuzzy topologies. Fuzzy Sets Syst. 210, 63-68 (2013)

27. Zadeh, L.A.: Similarity relations and fuzzy orderings. Inf. Sci. 3, 177-200 (1971)

28. Zeng, W., Li, H.: Inclusion measures, similarity measures, and the fuzziness of fuzzy sets and their relations. Int. J. Intell. Syst. 21(6), 639-653 (2006) 